\title{
Bacillus Licheniformis PF9 Improves Barrier Function and Alleviates Inflammatory Responses Against Enterotoxigenic Escherichia Coli F4 Infection in the Porcine Intestinal Epithelial Cells
}

Qiao Li

University of Manitoba https://orcid.org/0000-0002-7472-5333

Yanhong Chen

University of Manitoba

Changning Yu

University of Manitoba

Paula Azevedo

University of Manitoba

Joshua Gong

Agriculture and Agri-Food Canada

Chengbo Yang ( $\nabla$ chengbo.yang@umanitoba.ca )

University of Manitoba https://orcid.org/0000-0003-4449-5132

\section{Research}

Keywords: Bacillus licheniformis PF9, barrier function, inflammatory response, ETEC F4, IPEC-J2

Posted Date: June 4th, 2021

DOI: https://doi.org/10.21203/rs.3.rs-560501/v1

License: (c) (i) This work is licensed under a Creative Commons Attribution 4.0 International License.

Read Full License 


\section{Abstract}

Background: Enterotoxigenic Escherichia coli (ETEC) F4 commonly colonizes the small intestine and releases enterotoxins that impair the intestinal barrier function and trigger inflammatory responses. Although Bacillus licheniformis ( $B$. licheniformis) has been reported to enhance intestinal health, it remains to be seen whether there is a functional role of $B$. licheniformis in intestinal inflammatory response in intestinal porcine epithelial cell line (IPEC-J2) when stimulated with ETEC F4.

Methods: In the present study, the effects of $B$. licheniformis $P F 9$ on the release of pro-inflammation cytokines, cell integrity and nuclear factor-KB (NF-KB) activation were evaluated in ETEC F4-induced IPECJ2 cells.

Results: $B$. licheniformis PF9 treatment was capable of remarkably attenuating the expression levels of inflammation cytokines tumor necrosis factor-a (TNF-a), interleukin (IL)-8, and IL-6 during ETEC F4 infection. Furthermore, the gene expression of Toll-like receptor 4 (TLR4)-mediated upstream related genes of NF-KB signaling pathway has been significantly inhibited. These changes were accompanied by a significant decreased phosphorylation of p65 NF-KB during ETEC F4 infection with $B$. licheniformis PF9 treatment. The immunofluorescence and western blot analysis revealed that $B$. licheniformis $P F 9$ increased the expression levels of zona occludens 1 (ZO-1) and occludin (OCLN) in ETEC F4-infected IPEC-J2 cells. Meanwhile, the $B$. licheniformis PF9 could alleviate the epithelial barrier function assessed by the trans-epithelial electrical resistance (TEER) and cell permeability assay. Interestingly, $B$. licheniformis PF9 protect IPEC-J2 cells against ETEC F4 infection by decreasing the gene expressions of virulence-related factors (including luxS, estA, estB, and elt) in ETEC F4.

Conclusions: Collectively, our results suggest that $B$. licheniformis PF9 might reduce inflammation-related cytokines through blocking the NF-KB signaling pathways. Besides, B. licheniformis PF9 displayed a significant role in the enhancement of IPEC-J2 cell integrity.

\section{Background}

The intestine is not only a site of nutrient digestion, absorption and metabolism, but also an important defense line against the invasion of pathogenic agents in the external environment of the gut lumen. Intestinal epithelial cells (IECs) are continuously lined monolayer cells, which play an important role in this defense [1]. Increase of pathogenic bacteria in IECs can influence intestinal barrier function and change the structure of microbiota, leading to inflammatory reaction [2,3]. In particular, the disruption of barrier function causes damage to immune homeostasis and increases gut diseases such as inflammatory bowel diseases and diarrhea $[3,4]$. Therefore, the maintenance of the normal barrier function of IECs contributes to the gut homeostasis and health in human and animals.

Enterotoxigenic Escherichia coli (ETEC) is a common enteric pathogen causing intestinal disorder and diarrhea, which is one of the major challenges for human and animals, especially in pigs [4-6]. In human, the clinical symptoms of ETEC infections can range from mild diarrhea to a severe cholera-like syndrome, 
which seriously threatened the health of people [7]. In pigs, the ETEC-induced diarrhea can reduce growth performance as well as increase morbidity and mortality in post-weaning piglets, resulting in considerable economic losses to the swine industry $[8,9]$. The swine industry has largely relied on the prophylactic use of antibiotics to control ETEC and its associated diarrhea in the past [10]. However, there is a growing public concern over this practice due to the widespread of antibiotic resistance in zoonotic bacterial pathogens, which poses a threat to public health. Furthermore, the World Health Organization has strictly restricted the use of antibiotics in food-producing animals since 2017 [11]. Therefore, non-antibiotic strategies and technologies to control the pathogen using a natural alternative to antibiotics are urgently required to reduce their use in swine production.

The intestinal mucosal barrier plays a major role in maintaining the homeostasis of the gut microenvironment. Porcine intestinal epithelial cells (IPEC-J2) are recognized as a cell model for detecting intestinal inflammation and barrier function [12]. The integrity and regular permeability of tight junctions (TJs) were negatively influenced in IPEC-J2 cells with invasion of ETEC [13]. Furthermore, ETEC infection could increase the permeability of TJs in early weaned piglets [14]. Probiotics have been defined as living organisms that developed commercially for human uses, primarily as novel foods or dietary supplements, and in animal feeds for the prevention of gastrointestinal infections, with extensive use in the swine industries [15]. Probiotics have the beneficial effects of maintaining the normal intestinal milieu, modulating the immune system, and producing metabolites required for intestinal health [16]. Specific strains of probiotics have some potential to maintain intestinal barrier function and to reduce cell damage after being impaired by cytokines, chemicals, or pathogens [17]. Probiotic Enterococcus faecium NCIMB 10415 prevented the cytokine-induced dysfunction of epithelial barrier with pathogenic enteropathogenic or ETEC treatment in porcine (IPEC-J2) and human (Caco-2) intestinal cells [18]. Lactobacillus plantarum strengthened intestinal barrier function and tight junction integrity after ETEC challenge in IPEC-J2 cells [19]. Bacillus licheniformis (B. licheniformis), one of the most important bacteria, is used to inhibit pathogens, ameliorate intestinal flora homeostasis, and improve the nutritional value of animal feeds $[20,21]$. Research demonstrated that $B$. licheniformis YB9 significantly repaired the dysbiosis of intestinal flora in mice, characterized by decreasing the abundance of the potentially harmful bacteria Turicibacter [20]. Oral administration of a selected mixture of $B$. licheniformis BL1721, Lactobacillus johnsonii $L 531$ and $B$. subtilis BS1715 could alleviate inflammation and maintain mucosal barrier integrity in the ileum of pigs challenged with salmonella [22]. Another research showed that oral administration of the probiotic mix, i.e., B. licheniformis and B. subtilis could partially ameliorate E. coliinduced enteritis through preventing loss of intestinal epithelial barrier integrity via elevating zonula occludens-1 (ZO-1) expression in weaned pigs [23]. Therefore, it seems clear that the probiotic, $B$. licheniformis has a great potential application value for preventing pathogenic bacteria infections and maintaining healthy gut.

In our study, a strain of $B$. licheniformis PF9 was obtained from the Guelph Food Research Centre, Agriculture and Agri-Food Canada. We hypothesized that the probiotic B. licheniformis PF9 influenced the expression of tight junction proteins and alleviates inflammatory reactions in IPEC-J2 cells that contributed to a protective effect against ETEC F4-induced damage. Therefore, our study aims to explore 
the protective role of $B$. licheniformis PF9 in improving barrier disruption and inflammatory responses when pathogenic invasion occurs in the intestinal epithelial cells.

\section{Materials And Methods}

\section{Materials}

Dulbecco's modified Eagle's medium nutrient mix F12 (DMEM/F12), fetal bovine serum (FBS), doubleantibiotics (containing $100 \mathrm{U} / \mathrm{mL}$ penicillin and $100 \mathrm{U} / \mathrm{mL}$ streptomycin), and epidermal growth factor (EGF) were from Invitrogen (Fisher Scientific, Ottawa, ON, Canada).

\section{Cell culture}

IPEC-J2 cells (ACC 701, RRID: CVCL_2246, DSMZ-German Collection of Microorganisms) were cultured in DMEM/F12 culture medium supplemented with $5 \% \mathrm{FBS}, 1 \%$ double-antibiotics and $3 \mathrm{ng} / \mathrm{mL}$ EGF in the humidified incubator (Thermo Electron Corporation) with atmosphere of $5 \% \mathrm{CO}_{2}$ at $37^{\circ} \mathrm{C}$. The culture medium was replaced every two days. After growing to $80-90 \%$ confluence, the cells were digested with $0.25 \%$ trypsin and inoculated into cell culture plates for further experiments.

\section{Bacterial strains}

The strain of ETEC F4 was isolated from feces of piglets infected with post-weaning diarrhea in the Veterinary Diagnostic Services Laboratory, Government of Manitoba, Canada. The ETEC F4 strain was preserved in the $2 \mathrm{~mL}$ cryovials including cryopreservative-added broth. ETEC F4 was first incubated in Tryptic soy broth (TSB, Millipore Sigma) at $37^{\circ} \mathrm{C}$ for $16-18 \mathrm{~h}$. After dilution with fresh medium, the cultures were incubated under the same condition until the appearance of logarithmic phase. The optical density (OD) value at $600 \mathrm{~nm}$ was measured by spectrophotometer (Biochrom ${ }^{\mathrm{TM}}$, Fisher scientific). The OD values around 0.3-0.5 were used for the following experiments.

The strain of $B$. licheniformis PF9 was obtained from the Guelph Food Research Centre, Agriculture and Agri-Food Canada and preserved in sterile glycerol (10-20\%). The B. licheniformis PF9 strain was isolated from feces of piglets and incubated in TSB at $37^{\circ} \mathrm{C}$ overnight (about $16 \mathrm{~h}$ ). The OD values around 0.81.0 were used for the following experiments.

\section{Experimental design}

IPEC-J2 cells were detached with $0.25 \%$ trypsin (GibcoTM, Thermo fisher scientific) and diluted to $4 \times 10^{5}$ cells/mL. IPEC-J2 cells were then inoculated into different well plates and cultured in DMEM/F12 medium for about 2-3 days until growing to $80-90 \%$ confluence. For different treatments, the control group was cultured in DMEM/F12 medium without treatment, the ETEC group was treated with ETEC F4 $\left(10^{6}\right.$ $\mathrm{CFU} / \mathrm{mL}$ ) for $3 \mathrm{~h}$, the ETEC $+\mathrm{BL}$ group was pretreated the selected concentrations of $B$. licheniformis $P F 9$ for $2 \mathrm{~h}$ prior to both ETEC F4 and $B$. licheniformis $P F 9$ treatment together $(1: 5,1: 10,1: 25,1: 50$, or 1:100) 
for $3 \mathrm{~h}$, and the BL group was pretreated the selected concentrations of $B$. licheniformis PF9 for $2 \mathrm{~h}$ prior to its alone treatment $(3 \mathrm{~h})$.

\section{Lactate dehydrogenase (LDH) assay}

$\mathrm{LDH}$, a cytosolic enzyme present in many different cell types, will be released into the surrounding cell culture media when there is a damage to cells. IPEC-J2 cells were detached with $0.25 \%$ trypsin and diluted to $4 \times 10^{5}$ cells $/ \mathrm{mL}$. IPEC-J2 cells were then inoculated into 24 well plate at $2 \times 10^{5}$ cells $/$ well and cultured in DMEM/F12 medium for 3 days. After the previously described treatments, the supernatants containing released LDH of cultured IPEC-J2 cells were collected to determine the integrity of cells. After centrifugation at $12,000 \mathrm{r} / \mathrm{min}$ for $5 \mathrm{~min}$ at $4^{\circ} \mathrm{C}$, supernatants were carefully collected and transferred into new tubes. The supernatants of each sample $(50 \mu \mathrm{L})$ were added into a 96-well plate and the reaction mix for the assay was prepared by using LDH assay kit (CyQUANTTM, Thermo fisher scientific) according to the manufacturer's instructions. The absorbance at $490 \mathrm{~nm}$ and $680 \mathrm{~nm}$ was immediately measured by using a Synergy H4 hybrid multi-mode microplate reader (BioTek, Winooski, VT, USA). The activity of LDH was presented as a percentage of the LDH activity by the control group.

\section{Cytokine measurement by ELISA}

The IL-8 concentrations of culture supernatants were measured by ELISA kit (Invitrogen, Fisher Scientific) following the manufacturer's instructions. Briefly, $100 \mu \mathrm{L}$ of culture supernatant was used for IL-8 assay. At the end of the reaction process, the plates were read at $450 \mathrm{~nm}$, using a Synergy $\mathrm{H} 4$ hybrid multi-mode microplate reader (BioTek, Winooski, VT, USA).

\section{Quantitative PCR (qPCR)}

IPEC-J2 cells were seeded with a density of $5 \times 10^{5}$ cells per well in 12-well culture plates. After different treatments, total RNA was extracted by using Trizol (Invitrogen, Carlsbad, CA, U.S.) according to the manufacturer's instructions. The total RNA was dissolved in $20 \mu \mathrm{L}$ RNase-free water and stored at $-80^{\circ} \mathrm{C}$. The RNA concentration was determined using a NanoDrop 2000 Spectrophotometer (Nano-Drop Technologies, Wilmington, DE, U.S.) with purity ascertained (A260/A280) between 1.8 and 2.0. About $1 \mu \mathrm{g}$ total RNA from each sample was converted into cDNA by using iScript cDNA Synthesis Kit (Bio-Rad) according to the manufacturer's instruction. The primers were designed with Primer-Blast (https://www.ncbi.nlm.nih.gov/tools/primer-blast/index.cgi?LINK_LOC=BlastHome) and synthesized by Integrated DNA Technologies, Inc. The qPCR was performed to quantify the target genes, such as cytokines genes, barrier function genes, toll-like receptors genes, NF-kB signaling pathway genes, and virulence-related genes (Supplemental Tables S1 and S2). The cyclophilin-A (CycA) gene was used as the housekeeping gene in IPEC-J2 cells. The glyceraldehyde-3-phosphate dehydrogenase A (gapA) gene was used as the housekeeping gene in ETEC F4. The relative changes in gene expression levels in IPEC-J2 cells or ETEC F4 normalized against CyCA or gapA were determined by using the $2^{-\Delta \Delta C T}$ method [24].

\section{Immunofluorescent Staining}


Cells cultured onto cover-slips (Fisher Scientific) were fixed with 4\% paraformaldehyde for 20 mins and permeabilized with $0.3 \%$ Triton X-100 in PBS for 20 min. The cells were blocked with $5 \%$ goat serum (Jackson ImmunoResearch Laboratories, West Grove, PA, USA) for $1 \mathrm{~h}$ and then incubated with anti-rabbit polyclonal antibodies of zona occludens 1 (ZO-1, 1:100 dilution in PBS) and occludin (OCLN, 1:100 dilution, Thermal Scientific) at $4^{\circ} \mathrm{C}$ for $16 \mathrm{~h}$, respectively. The cells were then washed 3 times with PBS and incubated with an Alexa Fluor 488 goat anti-rabbit (green) or Alexa Fluor 584 goat anti-rabbit (red) antibody (Thermal Scientific, catalogue no. A-11034 or A-11037) for $1 \mathrm{~h}$ at $22^{\circ} \mathrm{C}$. For F-actin staining, the cells were incubated with phalloidin, CF488A (1:40 dilution in PBS, Biotium, Inc., Fremont, CA 94538, USA) at $22^{\circ} \mathrm{C}$ for $1 \mathrm{~h}$. Rinsed cells were mounted with Vectashield mounting medium with 4,6-Diamidino-2phenylindole (DAPI, Vector Laboratories, Inc. Burlingame, CA, USA). Images were taken by a Zeiss fluorescence microscope (Car-Zeiss Ltd., Toronto ON, Canada).

\section{Transepithelial electric resistance (TEER) and cell permeability measurement}

The IPEC-J2 cells with a density of $5 \times 10^{5}$ cells per well were seeded into Millicell membrane cell inserts (12 wells, Corning Costar). The TEER of cell monolayers was measured using a Millicell Electrical Resistance System (ESR-2, Millipore-Sigma). The TEER was monitored every two days until a monolayer of cells was completely differentiated. The TEER of four treatment groups (control, ETEC F4 alone, ETEC $F 4$ plus $B$. licheniformis $P F 9$, and $B$. licheniformis $P F 9$ alone) was measured before $(0 \mathrm{~h})$ and after ETEC F4 treatments $(1,2,3,4$, and $5 \mathrm{~h})$, respectively. The TEER of monolayers with/without adding ETEC F4 represented the ETEC F4 group and control group, respectively. The ETEC F4 plus $B$. licheniformis PFO group were pre-treated with $B$. licheniformis PF9 for $2 \mathrm{~h}$ and then incubated with ETEC F4 plus $B$. licheniformis PF9 for $3 \mathrm{~h}$. The value of TEER was corrected for background resistance by subtracting the contribution of cell free filter and the medium $(200 \Omega)$.

To quantify the paracellular permeability of cell monolayers, $0.5 \mathrm{mg} / \mathrm{mL}$ of $4 \mathrm{kDa}$ FITC-dextran (FD4, Sigma-Aldrich) was added to the apical side of the inserts. The basolateral medium aliquots were taken after $48 \mathrm{~h}$ of incubation. The diffused fluorescent tracer was then measured by fluorometry (excitation, $485 \mathrm{~nm}$, emission, $528 \mathrm{~nm}$ ) using a microplate reader (BioTek, Winooski, VT, USA). Measurements were performed in quadruplicate.

\section{Western blot}

Proteins from IPEC-J2 were extracted by using RIPA Lysate Buffer (Sigma-Aldrich) and their concentrations were determined by the BCA Protein Assay Kit according to the manufacturer's protocol (Fisher Scientific). Proteins were resolved by SDS-PAGE and transferred to a PVDF membrane using a Trans-Blot ${ }^{\circledR}$ Turbo $^{\mathrm{TM}}$ transfer system (Bio-Rad, Hercules, CA, USA). The membrane was then blocked for 2 $\mathrm{h}$ with $5 \%$ skim milk in Tris-buffered saline (TBS) at $22^{\circ} \mathrm{C}$, and was subsequently immunolabeled in primary antibodies diluted in TBS at $4^{\circ} \mathrm{C}$ for $16 \mathrm{~h}$. Primary antibodies to rabbit polyclonal anti-ZO-1 (1:1000 dilution), anti-OCLN (1:1000 dilution), anti-claudin-3 (1:1000 dilution) and anti-NF-KB p65 (1:1000 dilution), rabbit monoclonal anti-NF-KB phospho-p65 (1:1000 dilution) and mouse monoclonal anti- $\beta$ - 
actin (1:5000 dilution) from Invitrogen by Thermo Fisher scientific were used, respectively. After washing for $3 \times 10$ min with TBST, the blots were incubated with horseradish peroxidase-conjugated goat antimouse (1:350 dilution) or goat anti-rabbit IgG (1:4000 dilution) as a secondary antibody for 1 hour at $22^{\circ} \mathrm{C}$, respectively. Visualization of the antigen-antibody complex was conducted with a Clarity Max ECL Western Blotting Substrate (Bio-Rad) and immunoreactive proteins were visualized using the ChemiDoc ${ }^{\mathrm{TM}}$ MP imaging system (2.4.0.03, Bio-Rad). The protein bands were analyzed by Image Lab 6.0 software (Bio-Rad). The $\beta$-actin was used as the internal control. Values of target proteins were represented as the ratio of the optical density of the protein bands to the density of the respective $\beta$-actin band. The value of the normalized phosphorylation of p 65 protein was represented as the ratio of the density of phosphor-p65 to that of p65.

\section{Statistical analysis}

Data were presented as means \pm standard deviations (SD). Statistical analysis was performed using GraphPad Prism 7 (GraphPad Software, La Jolla, CA, USA). Differences between means were evaluated by one-way ANOVA. Tukey's multiple comparisons test was used. Difference of the mRNA abundance of virulence-related factors in ETEC F4 between ETEC and ETEC+BL groups were evaluated by unpaired $t$ test. Level of significance was set at $P<0.05$.

\section{Results}

\section{B. licheniformis PF9 protected IPEC-J2 cells against the ETEC F4-induced cytotoxicity}

To quantify the effect of $B$. licheniformis PF9 protected IPEC-J2 cells against the ETEC F4, the cytotoxicity was measured by LDH activity in the supernatants. As shown in Fig. 1, the ETEC group showed that ETEC F4 challenge at $10^{6} \mathrm{CFU} / \mathrm{mL}$ significantly induced the toxicity effect in IPEC-J2 cells $(P<0.05)$. However, this cytotoxicity could be remarkably attenuated in the ETEC + BL groups, in which, ETEC F4 was simultaneously added with $B$. licheniformis $P F 9$ at the ratios of 1:5, 1:10, 1:25, 1:50, or 1:100 when compared with that in the ETEC group $(P<0.05)$. Meanwhile, the result of the BL group demonstrated that the cells treated with the highest concentration of $B$. licheniformis $P F 9\left(10^{8}\right)$ did not significantly increase LDH release compared with control group $(P>0.05)$. These results indicated that the treatment with $B$. licheniformis PF9 maintains the integrity of the cellular membrane in IPEC-J2 cells.

\section{Effect of B. licheniformis PF9 on ETEC F4-induced the alteration of cytokine IL-8 secretion in IPEC-J2 cells}

The effects of $B$. licheniformis PF9 on cytokine IL-8 secretion changes in IPEC-J2 cells challenged with ETEC F4 were detected by ELISA. As shown in Fig. 2, the result of the BL group demonstrated that the cells treated with the highest concentration of $B$. licheniformis PF9 $\left(10^{8}\right)$ had no effect on the IL-8 secretion compared with control group $(P>0.05)$. However, the ETEC group showed that ETEC F4 challenge at $10^{6} \mathrm{CFU} / \mathrm{mL}$ significantly stimulated the IL-8 secretion in IPEC-J2 cells $(P<0.05)$.

Interestingly, this change could be markedly diminished under the treatment of adding both ETEC F4 and 
B. licheniformis PF9 at the ratios of $1: 5,1: 10,1: 25,1: 50$, or 1:100 $(P<0.05)$. However, this effect seemed not significant with the higher ratios of ETEC F4 and $B$. licheniformis $P F 9$, i.e., dilutions ratios over 1:10. Therefore, we chose the ratio of ETEC F4 and $B$. licheniformis PF9 at 1:10 (i.e., $10^{6} \mathrm{CFU} / \mathrm{mL}$ ETEC F4: $10^{7}$ $\mathrm{CFU} / \mathrm{mL}$ B. licheniformis PF9) in the following experiment to explore the effect of Bacillus licheniformis PF9 against ETEC F4-induced barrier function disruption and inflammatory responses in the IPEC-J2 cells.

\section{Effect of B. licheniformis PF9 on ETEC F4-induced gene expression of inflammatory cytokines}

In order to investigate the effects of $B$. licheniformis PF9 on expression profiles of inflammatory cytokines on IPEC-J2 cells subjected to ETEC F4 challenge, the mRNA expressions of pro-inflammatory cytokines (TNF-a, IL-8, and IL-6) were followed by RT-PCR. As shown in Fig. 3, it was demonstrated that $B$. licheniformis PF9 $\left(10^{7} \mathrm{CFU} / \mathrm{mL}\right)$ alone didn't show differences on the mRNA expression of TNF- $\mathrm{a}$, IL-8, and IL- 6 when compared with the control group $(P>0.05)$. However, it was shown that ETEC F4 challenge at $10^{6} \mathrm{CFU} / \mathrm{mL}$ significantly stimulated the inflammatory gene expressions of TNF-a, IL-8, and IL-6 ( $P<$ 0.05). It is worth noticing that these up-regulations could be markedly decreased under the treatment of adding both ETEC F4 and $B$. licheniformis PF9 at the ratio of 1:10 $(P<0.05)$. Nevertheless, the gene expressions of TNF- $a$ and IL-8 in the ETEC + BL group were still significantly higher than those in the control group (Figs. $3 a$ and $b, P<0.05$ ). The gene expressions of IL- 6 in the ETEC $+B L$ group showed no difference than those in the control group (Figs. $3 c, P<0.05$ ).

\section{Protective effect of B. licheniformis PF9 against ETEC F4-induced morphological changes of cytoskeleton and tight junctions (TJs) in IPEC-J2 cells}

The effects of $B$. licheniformis PF9 and pathogen ETEC F4 on the distribution of cytoskeleton (F-actin) as well as TJs (zonula occludes 1, ZO-1 and occludin, OCLN) were evaluated by immunofluorescence. $B$. licheniformis PF9 treatment alone did not alter the expression of cytoskeleton F-actin. The cell morphology displayed disordered and the fluorescence of F-actin seemed to be unclear and even disappeared in some cells after ETEC F4 infection. In comparison, the $B$. licheniformis PF9 treatment notably maintained normal morphology and increased the green fluorescent spots against pathogenic infection in the cytoskeleton of F-actin (Fig. 4a). Meanwhile, there were highly similar morphological changes of TJs proteins. TJs proteins of ZO-1 and OCLN did not show the obvious change after $B$. licheniformis PF9 treatment alone, which were present at the apical intercellular borders in a belt-like manner, encircling the cells and delineating the cellular borders. The fluorescence was discontinuous and unclear in cells infected with ETEC F4. However, B. licheniformis PF9 was able to ameliorate the abnormal morphology of junction proteins, whereas the green (ZO-1) or red (OCLN) spots were increased compared with ETEC F4 infection (Figs. 4b and 4c).

Influence of B. licheniformis PF9 on ETEC F4-induced gene and protein expressions of tight junctions (TJs) in IPEC-J2 cells 
To investigate the possible membrane barrier disruption caused by ETEC F4 and the potential role of $B$. licheniformis PF9 in restoring such damage, the gene and protein expressions of TJs were performed (Fig. 5). The effects of treatment on the abundance of transcripts of ZO-1, OCLN, claudin-1 (CLDN1) and claudin-3 (CLDN3) were examined in IPEC-J2 cells. There were no significant differences in the relative abundance of three transcripts (ZO-1, CLDN1 and CLDN3) in the four different treatments (Fig. 5a, P > 0.05 ). However, the abundance of mRNA OCLN was significantly reduced after challenge with ETEC F4 ( $P$ $<0.05)$, and the treatment with $B$. licheniformis PF9 could reverse this change when compared with the control cells.

Western blotting was used to verify treatment differences in expression of TJ components (ZO-1, OCLN and CLDN3) at the protein level in IPEC-J2 cells (Fig. 5b). From the results, it was found that $B$. licheniformis PF9 did not influence the proteins expressions of ZO-1, OCLN and CLDN3. Meanwhile, in agreement with the mRNA expression of CLDN3, there were no changes of protein abundance in the four different groups (Fig. 5b, $P>0.05$ ). However, ETEC F4 challenge was able to obviously decrease expression levels of ZO-1 and OCLN in IPEC-J2 cells $(P<0.05)$ while concurrent treatment ETEC F4 with $B$. licheniformis $P F 9$ had the capability to significantly increase the levels of these two TJ proteins (Fig. 5b, $\mathrm{P}<0.05$ ).

\section{Protective effect of B. licheniformis PF9 against ETEC F4-induced damage to barrier integrity}

The epithelial barrier function of IPEC-J2 cells monolayer was assessed by TEER and cell permeability in response to ETEC F4 infection in the absence or presence of $B$. licheniformis PF9. As shown in Fig. 6a, single $B$. licheniformis PF9 treatment had no negative effect on the TEER in IPEC-J2 cells, however, the percentage of relative TEER values significant decreased (about $65 \%$ and $70 \%$, respectively) in cells infected with ETEC F4 at $4 \mathrm{~h}$ and $5 \mathrm{~h}(P<0.05)$. When cells were pre-treated with $B$. licheniformis $P F 9$ followed by challenge with ETEC F4, however, the reductions of TEER were less (about $30 \%$ and $40 \%$, respectively) at $4 \mathrm{~h}$ and $5 \mathrm{~h}$. Consistently, a significant augmentation of fluorescence intensity observed in the ETEC F4 challenge was substantially minimized after $B$. licheniformis PF9 supplementation (Fig. 6b, $P<0.05)$. These results indicated that pre-treatment with $B$. licheniformis $P F 9$, while itself having no disadvantageous effect on the barrier integrity of IPEC-J2 cells, did confer some protection on barrier integrity when cells were subsequently challenged with ETEC F4.

\section{Effect of B. licheniformis PF9 on the mRNA abundance of virulence-related factors in ETEC F4}

To determine the underlying mechanism of $B$. licheniformis PF9 in protecting IPEC-J2 cells against ETEC F4 infection, the gene expressions of virulence-related factors (including faeG, luxS, estA, estB, and elt) of ETEC F4 were examined in the presence and absence of $B$. licheniformis PF9. The quantitative PCR analysis revealed that co-cultured with $B$. licheniformis $P F 9$ was able to downregulate the expression of bacterial genes related to quorum sensing (IuxS), as well as STs (estA and estB) and LT (elt) toxin secretion in ETEC F4-infected IPEC-J2 cells (Figs. 7a, and c-e, $P<0.05$ ). However, the faeG gene expressions related to fimbrial biosynthesis of ETEC F4 strains did not show obvious difference in the 
two groups (Figs. $7 \mathrm{~b}, \mathrm{P}>0.05$ ). These results suggest that $B$. licheniformis $P F 9$ was involved in alleviating ETEC F4-induced cytotoxicity by decreasing the expressions of virulence-related factors in IPEC-J2 cells.

\section{The functions of NF-KB involved in TLRs-triggered signaling pathways during the process of $\mathrm{B}$. licheniformis PF9 regulating the inflammatory response}

To explore the underlying mechanism of ETEC F4-induced inflammation, the mRNA expression of toll-like receptors (TLR2, TLR4, TLR5, and TLR7) were measured by RT-PCR. The gene expression of TLR2 and TLR4 was significantly higher $(P<0.05)$ in the ETEC F4 group, but this increase in gene expression could be attenuated by the $B$. licheniformis PF9 treatment. On the other hand, the levels of TLR5 and TLR7 mRNAs did not change in the ETEC group compared to the control group (Fig. 8a, P > 0.05). Furthermore, compared with the control group, the ETEC F4 increased the mRNA expressions of the related upstream genes of the NF-KB signaling pathway (MyD88 and TAK1). While the mRNA levels of TRAF6 and IRAK did not show significant increase in the ECET F4-induced cells. Meanwhile, $B$. licheniformis $P F 9$ treatment significantly blocked the increase in MyD88, TRAF6, IRAK and TAK1 mRNA levels (Fig. 8b, P<0.05). The stimulation of NF-KB signaling pathway eventually led to the increase of phosphorylated p65 protein, while $B$. licheniformis PF9 could reverse this change (Fig. $8 c, P<0.05$ ).

\section{Discussion}

The intestine and associated epithelium not only can effectively digest and absorb nutrients, but it is also armed with a large number of immune cells ready to intervene when pathogenic bacteria breach the intestinal epithelium. Gut disorders and dysfunctions might induce gut diseases such as inflammatory bowel diseases and diarrhea, leading to be harmful to animal health and growth performance [25]. In fact, the intestinal epithelial cells (IECs) play important roles in the physical defense [26]. The maintenance of the barrier function of IECs contributes to the gut homeostasis and health. Therefore, nutritional and/or pharmacological interventions aimed at reinforcing this barrier are being actively sought after for human and animal health.

ETEC is a common enteric pathogen form colibacillosis causing intestinal disorder and diarrhea, which is one of the major challenges for human and several species of farm animals [27, 28]. ETEC are characterized by producing virulence factors such as adhesins and enterotoxins. The most common adhesins that promote binding and colonization of the intestinal epithelium are expressed in the fimbriae, such as the F4 (also designated K88), F5 (K99), F6 (987P), F17 and F18. Once established in the animal small intestine, ETEC produces enterotoxins, namely labile toxins (LTs) and stable toxins (STs), acting on enterocytes and resulting in diarrhea [29]. In the livestock industry, where the prophylactic use of antibiotics in ETEC infections is being restricted in most countries, an increasing number of antibiotic alternatives are being developed to alleviate the intestinal inflammation and protect digestive health in the gut [30]. Probiotics, an attractive group of such alternatives, can provide beneficial health effects to the host by excluding pathogenic bacteria, producing bactericidal agents, regulating immune response and maintaining gut barrier integrity [31, 32]. B. licheniformis, gram-positive bacteria, has been popularly 
considered as a beneficial probiotic bacterium [33]. A recent study showed that $B$. licheniformis $C K 1$ could alleviate the toxic effects of zearalenone in feed on weaned female Tibetan piglets [34]. The ileum microbiota disorder caused by necrotic enteritis was normalized by dietary $B$. licheniformis supplementation in chickens [35]. A recent research also showed the combination of B. subtilis B21 and $B$. licheniformis $B 26$ improved the intestinal health and mucosal barrier of broiler chickens by the regulation of tight junction proteins in chickens with Clostridium perfringens-induced necrotic enteritis [36]. Meanwhile, oral administration of $B$. licheniformis and $B$. subtilis mixture ameliorated ETEC F4induced enteritis in the weaned F4ab/acR ${ }^{-}$(ETEC F4ab/ac receptor negtive) pigs [37]. Furthermore, $B$. licheniformis was found to have its anti-inflammatory actions by significantly intervening with the secretion of IL-8 in IPEC-J2 [38]. Although B. licheniformis strain is especially popular used as an additive in processed feed, the mode of action to protect the gut health from ETEC-induced disruption is not wellunderstood. In the present study, ETEC F4 bacteria, isolated from feces of piglets infected with postweaning diarrhea in the Veterinary Diagnostic Services Laboratory from the Government of Manitoba in Canada, was used to establish the model of harmful bacteria invasion in vitro. A previous study from this laboratory, showed that this strain of ETEC F4 can affect the nutrient absorption, immunity and gut barrier function in weaned piglets [39]. It was hypothesized that $B$. licheniformis $P F 9$ isolated from feces of piglets in this study may be involved in attenuating/ ameliorating ETEC-induced changes in mucosal barrier function and the inflammatory responses of intestinal epithelial cells.

$\mathrm{LDH}$, an oxidoreductase enzyme, is found in the cytoplasm of many different cells. Previous research showed that both intracellular and extracellular stress can lead to an impaired mucosal barrier function and subsequently stimulate release of LDH from cells [40]. Thus, the amount of LDH detected in the culture medium is generally used as an indicator for determining damaged cells. In the present study, we found that ETEC F4 challenge at $10^{6} \mathrm{CFU} / \mathrm{mL}$ was able to induce a significant increase in LDH release by IPEC-J2 cells. It's noteworthy to notice though that the addition of both ETEC F4 and B. licheniformis PF9 together at the ratios of $1: 5,1: 10,1: 25,1: 50$, or $1: 100$ to the culture medium markedly decreased LDH release (Fig. 1), which confirmed an positive role of $B$. licheniformis PF9 in protecting the integrity of the cellular membrane in IPEC-J2 cells.

Cytokine production is considered as an important indicator in response to ETEC F4. Many $B$. licheniformis strains were proved to have the ability to alleviate inflammatory response in vitro and in vivo One study has demonstrated that $B$. licheniformis strain can significantly decrease production of the pro-inflammatory cytokines (IL-8) under the challenge of Salmonella enterica serovar Typhimurium in intestinal epithelial cells [38]. Pretreatment with Bacillus species spores (B. licheniformis, $B$. indicus, $B$. subtilis, $B$. clausii, and $B$. coagulans) lowered the serum levels of pro-inflammatory cytokines (TNF-a and $1 \mathrm{~L}-1 \beta$ ) against acetaminophen-induced acute liver injury in rats [41]. Similarly, we found that $B$. licheniformis $P F 9$ treated with pathogenic exposure at the ratios of $1: 10,1: 25,1: 50$, or 1:100 (ETEC F4 to B. licheniformis PF9) actually attenuated the release of cytokines IL-8 (Fig. 2). However, this antiinflammatory effect seemed not to cause any significant change with the higher ratio of ETEC F4 and $B$. licheniformis PF9 (over 1:10). Therefore, we ultimately chose the treatment with the ratio of ETEC F4 and 
B. licheniformis PF9 at 1:10 (i.e., $106 \mathrm{CFU} / \mathrm{mL}$ ETEC F4: $107 \mathrm{CFU} / \mathrm{mL}$ B. licheniformis $P F 9$ ) in the following experiment to explore the effect of Bacillus licheniformis PF9 against ETEC F4-induced barrier function disruption and inflammatory responses in the IPEC-J2 cells. Interestingly, it was shown that ETEC F4 challenge significantly stimulated the inflammatory gene expressions of TNF-a, IL-8, and IL-6, which can be markedly decreased under the treatment of adding both ETEC F4 and B. licheniformis PF9 at the ratio of 1:10 (Fig. 3). These results suggest that $B$. licheniformis $P F 9$ could down-regulate cytokines production, and probably slow down cell damage and relatively weakening inflammation.

Intestinal barrier plays an important role in organ development and disease pathogenesis. It is closely associated with functional TJs which are a dynamic complex locating between epithelial cells and play a key role in critical structure for paracellular permeability [42]. In this sense, alteration of this multifunctional and continuous assembled cells layer may contribute to disordering the normal paracellular permeability (ion, water and solutes transport) and epithelium integrity [43]. Occludin (OCLN), ZO-1 and claudins (CLDN1 and CLDN3) are crucial transmembrane proteins to maintain the physiological functions of TJs. Decreasing occludin and ZO-1 expression could increase the intestinal permeability in weaned piglets or IPEC-J2 $[12,44]$. The integrity and regular permeability of TJs were negatively influenced by invasion of ETEC F4 in IPEC-J2 cells [13]. Meanwhile, ETEC F4 infection could increase the permeability of TJs in early weaned piglets [45] and the probiotics mix of $B$. licheniformis and $B$. subtilis was proven to reduce ETEC F4-induced membrane barrier disruption by maintaining proper position of ZO-1 in weaned pigs [23]. These supported evidences were consistent with our presented data that ETEC F4 challenge could decrease the expression of ZO-1 and OCLN in IPEC-J2 cells, and these downregulations could be partially reversed by the application of $B$. licheniformis PF9 (Fig. 5). Moreover, immunofluorescence staining showed that $B$. licheniformis PF9 promoted the expression of F-actin, ZO-1 and OCLN in the cytomembrane (Fig. 4). Furthermore, TEER is usually considered as a good indicator to evaluate the integrity and tightness of intestinal epithelial barrier model $[12,46]$. It was demonstrated that the relative TEER and FD4 values did significantly decrease and increase, respectively, after ETEC F4 stimulation in IPEC-J2. However, these damages could be prevented by the treatment of $B$. licheniformis PF9 to some degree (Fig. 6). As a whole, these results indicated that $B$. licheniformis PF9 could enhance barrier functions in the IPEC-J2 cells. Therefore, in our study, B. licheniformis PF9-enhanced expression of TJ proteins seem to contribute to strengthening the epithelial barriers against bacterial pathogen infections.

Quorum sensing (QS) is a bacterial cell-cell communication process, regulating the expression of genes that affect a variety of cellular processes including bioluminescence, sporulation, biofilm formation, antibiotic production, and virulence factors secretion [47]. QS is based on production, secretion and response to small signaling molecules, called autoinducers (Al) [48]. Al-2, a universal autoinducer, is widely distributed in numerous Gram-positive and Gram-negative bacteria [49]. LuxS plays an important role in Al-2 biosynthesis, involving conversion of ribose-homocysteine into homocysteine (SRH) and 4,5dihydroxy-2,3pentanedione (DPD) $[49,50]$. It was reported that $B$. subtilis $R$-18 could inhibit QS-mediated virulence in Serratia marcescens [51]. In the present study, B. licheniformis PF9 could down-regulate the expression of bacterial genes related to quorum sensing (IUXS), as well as STs (estA and estB) and LT 
(elt) toxin secretion in ETEC F4-infected IPEC-J2 cells (Fig. 7). Therefore, B. licheniformis PF9 could be involved in alleviating ETEC F4-induced cytotoxicity by decreasing QS and virulence factors secretion in IPEC-J2 cells.

The earliest immune cells distinguishing self-molecules from extraneous structures is based on the conserved pathogen-associated molecular patterns (PAMPs) that are recognized by multiple classes of molecules called pattern recognition receptors (PRRs) [52]. Toll-like receptors (TLRs), a class of PRRs, are transmembrane proteins that are localized to the plasma or endosomal membranes of many types of cells. The activation of TLRs mediates pro-inflammatory signaling via the myeloid differentiation primary response protein (MYD88) and TIR domain-containing adapter molecule 1 (TRIF) pathways [53]. Research also found that TLR2/TLR4-mediated NF-KB signaling pathways have been involved in the ETEC-induced inflammation process in IPEC-J2 cells [54]. From our results, $B$. licheniformis PF9 could inhibit the inflammation process via TLR4-mediated NF-KB signaling pathways. Recently, links were established between inflammation and ETEC enterotoxins. A recent study has shown that LTs also can activate both NF-KB and MAPK signaling pathways, involved in ETEC adherence [55]. Most of the secreted LT is found to be associated with outer membrane vesicles (OMVs). LT-OMVs induce a great number of inflammatory cytokines as IL- 6 and TNF- $a$ through p38, ERK1/2 and NF-KB signaling pathways in T84 cells [56]. However, the relationship between enterotoxin and inflammation and its underlying mechanism on $B$. licheniformis PF9 resistance to ETEC infection need further research.

\section{Conclusions}

In conclusion, our data nominated that $B$. licheniformis PF9 might inhibit TNF-a, IL-8, and IL-6 production due to the inhibition of NF-KB signaling pathway. It might also be possible for $B$. licheniformis $P F 9$ to enhance the cell integrity according to the up-regulated expression of tight junction proteins. Our research provides data to advance the understanding of the underlying molecular mechanisms that probiotics could improve gut health by improving their inflammatory and intestinal barrier during ETEC infection at the cellular level. Furthermore, the probiotic effects on the pathogenic prevention in animal model would be emphasized in the future.

\section{Abbreviations}

ETEC: Enterotoxigenic Escherichia coli; B. licheniformis: Bacillus licheniformis; IPEC-J2: intestinal porcine epithelial cell line; NF-кB: nuclear factor-кB; TNF-a: tumor necrosis factor-a; IL: interleukin; TLR4: Toll-like receptor 4; ZO-1: zona occludens 1; OCLN: occluding; TEER: trans-epithelial electrical resistance; IECs: intestinal epithelial cells; TJs: tight junctions; DMEM/F12: Dulbecco's modified Eagle's medium nutrient mix F12; FBS: fetal bovine serum; EGF: epidermal growth factor; TSB: Tryptic soy broth; CycA: cyclophilinA; gapA: glyceraldehyde-3-phosphate dehydrogenase A; CLDN1: claudin-1; CLDN3: claudin-3; OD: optical density; LDH: lactate dehydrogenase; LTs: labile toxins; STs: stable toxins; QS: quorum sensing; PAMPs: pathogen-associated molecular patterns; PRRs: pattern recognition receptors; TLRs: toll-like receptors; TRIF: TIR domain-containing adapter molecule 1; outer membrane vesicles (OMVs). 


\section{Declarations}

\section{Acknowledgments}

Not applicable.

\section{Authors' Contributions}

Chengbo Yang conceived the study. Qiao Li and Chengbo Yang designed the experiments. Qiao Li, Yanhong Chen and Changning Yu performed the experiments. Qiao Li wrote the manuscript. Paula Azevedo and Joshua Gong improved the language. All authors reviewed the manuscript.

\section{Funding}

This work was supported by the Agriculture and Agri-Food Canada, AAFC's IOP project, Manitoba Pork and Swine Innovation Porc, and Canada Foundation for Innovation (CFI). Qiao Li was a Visiting Ph.D. student at the University of Manitoba, who was supported by the Chinese Scholarship Council (CSC).

\section{Availability of data and materials}

The data used to support the findings of this study are available from the corresponding author upon reasonable request.

\section{Ethics approval and consent to participate}

Not applicable.

\section{Consent for publication}

All the authors read and agree to the content of this paper and its publication.

\section{Competing interests}

All authors declare that they have no conflict of interest.

\section{Author details}

${ }^{1}$ Department of Animal Science, University of Manitoba, Winnipeg, Manitoba, Canada R3T 2N2

${ }^{2}$ College of Veterinary Medicine, Huazhong Agricultural University, Wuhan, China $430070{ }^{3}$ Guelph Research and Development Centre, Agriculture Agri-Food Canada, Guelph, Ontario, Canada N1G 5C9

\section{References}

1. Pitman RS, Blumberg RS. First line of defense: the role of the intestinal epithelium as an active component of the mucosal immune system. J Gastroenterol. 2000;35(11):805-14. 
2. Ahmed I, Roy BC, Khan SA, Septer S, Umar S. Microbiome, Metabolome and Inflammatory Bowel Disease. Microorganisms. 2016;4(2):20.

3. Landy J, Ronde E, English N, Clark SK, Hart AL, Knight SC, et al. Tight junctions in inflammatory bowel diseases and inflammatory bowel disease associated colorectal cancer. World $\mathrm{J}$ Gastroenterol. 2016;22(11):3117-26.

4. Lamberti LM, Bourgeois AL, Fischer Walker CL, Black RE, Sack D, Vinetz JM. Estimating diarrheal illness and deaths attributable to shigellae and Enterotoxigenic Escherichia coli among older children, adolescents, and adults in South Asia and Africa. PLoS Neglect Trop D. 2014;8(2):e2705.

5. Nagy B, Fekete PZ. Enterotoxigenic Escherichia coli (ETEC) in farm animals. Vet Res. 1999;30(23):259-84.

6. Nagy B, Fekete PZ. Enterotoxigenic Escherichia coli in veterinary medicine. Int J Med Microbiol. 2005;295(6-7):443-54.

7. Qadri FJCmr. Enterotoxigenic Escherichia coli in developing countries: epidemiology, microbiology, clinical features, treatment, and prevention. 2005;18.

8. Rhouma M, Fairbrother JM, Beaudry F, Letellier A. Post weaning diarrhea in pigs: risk factors and non-colistin-based control strategies. Acta Vet Scand. 2017;59(1):31.

9. Heo J, Opapeju F, Pluske J, Kim J, Hampson D, Nyachoti C. Gastrointestinal health and function in weaned pigs: a review of feeding strategies to control post-weaning diarrhoea without using in-feed antimicrobial compounds. J Anim Physiol An N. 2013;97(2):207-37.

10. Economou V, Gousia P. Agriculture and food animals as a source of antimicrobial-resistant bacteria. Infect Drug Resist. 2015;8:49.

11. Aidara-Kane A, Angulo FJ, Conly JM, Minato Y, Silbergeld EK, McEwen SA, et al. World Health Organization (WHO) guidelines on use of medically important antimicrobials in food-producing animals. Antimicrob Resist In. 2018;7(1):7.

12. Omonijo FA, Liu S, Hui Q, Zhang H, Lahaye L, Bodin J-C, et al. Thymol improves barrier function and attenuates inflammatory responses in porcine intestinal epithelial cells during lipopolysaccharide (LPS)-induced inflammation. J Agr Food Chem. 2018;67(2):615-24.

13. Yu H, Ding X, Shang L, Zeng X, Liu H, Li N, et al. Protective ability of biogenic antimicrobial peptide microcin J25 against Enterotoxigenic Escherichia Coli-induced intestinal epithelial dysfunction and inflammatory responses IPEC-J2 cells. Front Cell Infect Mi. 2018;8:242.

14. Yang KM, Jiang ZY, Zheng CT, Wang L, Yang XF. Effect of Lactobacillus plantarum on diarrhea and intestinal barrier function of young piglets challenged with enterotoxigenic Escherichia coli K88. J Anim Sci. 2014;92(4):1496-503.

15. Hong HA, Duc LH, Cutting SM. The use of bacterial spore formers as probiotics. FEMS Microbiol Rev. 2005;29(4):813-35.

16. Teitelbaum JE, Walker, Allan W. Nutritional impact of probiotics as protective gastrointestinal organisms. international seminars in pediatric gastroenterology nutrition. 2002. 
17. Ahrne S, Hagslatt ML. Effect of lactobacilli on paracellular permeability in the gut. Nutrients. 2011;3(1):104-17.

18. Shanti K, Angelika B, Holger M, Aschenbach JrR, Katharina B, Karsten T, et al. Enterococcus faecium NCIMB 10415 Modulates epithelial integrity, heat shock protein, and proinflammatory cytokine response in intestinal cells. Mediat Inflamm. 2014;2015:1-11.

19. Wu Y, Zhu C, Chen Z, Chen Z, Zhang W, Ma X, et al. Protective effects of Lactobacillus plantarum on epithelial barrier disruption caused by enterotoxigenic Escherichia coli in intestinal porcine epithelial cells - ScienceDirect. Vet Immunol Immunop. 2016;172:55-63.

20. Wang S, Hou Q, Guo Q, Zhang J, Shen L. Isolation and Characterization of a DeoxynivalenolDegrading Bacterium Bacillus licheniformis YB9 with the Capability of Modulating Intestinal Microbial Flora of Mice. Toxins. 2020;12(3).

21. Lan XR, Kim HI. Effects of Bacillus licheniformis and Bacillus subtilis complex on growth performance and faecal noxious gas emissions in growing-finishing pigs. J SCI Food Agr. 2018.

22. Liu X, Xia B, He T, Li D, Zhu YH. Oral Administration of a Select Mixture of Lactobacillus and Bacillus alleviates inflammation and maintains mucosal barrier integrity in the ileum of pigs challenged with Salmonella Infantis. Microorganisms. 2019;7(5):135-.

23. Yang GY, Zhu YH, Zhang W, Zhou D, Zhai CC, Wang JF. Influence of orally fed a select mixture of Bacillus probiotics on intestinal T-cell migration in weaned MUC4 resistant pigs following Escherichia coli challenge. Vet Res. 2016;47(1):71.

24. Livak KJ, Schmittgen TD. Analysis of relative gene expression data using real-time quantitative PCR and the $2-\Delta \Delta C T$ method. Methods. 2001;25(4):402-8.

25. Sartor RB. Mechanisms of disease: pathogenesis of Crohn's disease and ulcerative colitis. Nat clin Pract Gastr. 2006;3(7):390-407.

26. Allaire JM, Crowley SM, Law HT, Chang S-Y, Ko H-J, Vallance BA. The intestinal epithelium: central coordinator of mucosal immunity. Trends immunol. 2018;39(9):677-96.

27. Nagy B, Fekete PZ. Enterotoxigenic Escherichia coli (ETEC) in farm animals. 1999.

28. Qadri F, Svennerholm A-M, Faruque A, Sack RB. Enterotoxigenic Escherichia coli in developing countries: epidemiology, microbiology, clinical features, treatment, and prevention. Clin Microbiol Rev. 2005;18(3):465-83.

29. None. Animal enterotoxigenic escherichia coli. Ecosal Plus. 2016;7(1).

30. Ducatelle R, Goossens E, De Meyer F, Eeckhaut V, Antonissen G, Haesebrouck F, et al. Biomarkers for monitoring intestinal health in poultry: present status and future perspectives. Vet Res. 2018;49(1):1-9.

31. Walter J, Britton RA, Roos S. Host-microbial symbiosis in the vertebrate gastrointestinal tract and the Lactobacillus reuteri paradigm. P Natl A Sci. 2011;108(Supplement 1):4645-52.

32. Miyauchi E, O'Callaghan J, Buttó LF, Hurley G, Melgar S, Tanabe S, et al. Mechanism of protection of transepithelial barrier function by Lactobacillus salivarius: strain dependence and attenuation by 
bacteriocin production. Am J Physiol-Gastr L. 2012;303(9):G1029-G41.

33. Lee NK, Kim WS, Paik HD. Bacillus strains as human probiotics: characterization, safety, microbiome, and probiotic carrier. Food Sci Biotechnol. 2019;28(5).

34. Guanhua L, Wang L, Jeruei L, et al. Bacillus licheniformis CK1 alleviates the toxic effects of zearalenone in feed on weaned female Tibetan piglets. J Anim Sci. 2018.

35. Xu S, Lin Y, Zeng D, Zhou M, Zeng Y, Wang H, et al. Bacillus licheniformis normalize the ileum microbiota of chickens infected with necrotic enteritis. Rep. 2018;8(1):1744.

36. Musa BB, Duan Y, Khawar H, Sun Q, Ren Z, Mohamed MAE, et al. Bacillus subtilis B21 and Bacillus licheniformis B26 improve intestinal health and performance of broiler chickens with Clostridium perfringens-induced necrotic enteritis. J Anim Physiol An N. 2019;103.

37. Zhou D, Zhu YH, Zhang W, Wang ML, Fan WY, Song D, et al. Oral administration of a select mixture of Bacillus probiotics generates Tr1 cells in weaned F4ab/acR - pigs challenged with an F4 + ETEC/VTEC/EPEC strain. Vet Res. 2015.

38. Aperce CC, Burkey TE, Kukanich B, Crozierdodson BA, Dritz SS, Minton JE. Interaction of Bacillus species and Salmonella enterica serovar Typhimurium in immune or inflammatory signaling from swine intestinal epithelial cells. J Anim Sci. 2010;88(5):1649-56.

39. Choi J, Wang L, Liu S, Lu P, Zhao X, Liu H, et al. Effects of a microencapsulated formula of organic acids and essential oils on nutrient absorption, immunity, gut barrier function, and abundance of enterotoxigenic Escherichia coli F4 in weaned piglets challenged with E. coli F4. J Anim Sci. 2020;98(9):skaa259.

40. Koh JY, Choi DW. Quantitative determination of glutamate mediated cortical neuronal injury in cell culture by lactate dehydrogenase efflux assay. J Neurosci Meth. 1987;20(1):83-90.

41. Neag MA, Catinean A, Muntean DM, Pop MR, Bocsan Cl, Botan EC, et al. Probiotic Bacillus spores protect against acetaminophen induced acute liver injury in rats. Nutrients. 2020;12(3).

42. Yap A, Mullin J, Stevenson B. Molecular analyses of tight junction physiology: insights and paradoxes. J Membrane Biol. 1998;163(3):159-67.

43. Hammer AM, Morris NL, Earley ZM, Choudhry MA. The first line of defense: the effects of alcohol on post-burn intestinal barrier, immune cells, and microbiome. Alcohol Res-curr Rev. 2015;37(2):209.

44. Zhang B, Guo Y. Supplemental zinc reduced intestinal permeability by enhancing occludin and zonula occludens protein-1 (ZO-1) expression in weaning piglets. Br J Nutr. 2009;102(5):687-93.

45. McLamb BL, Gibson AJ, Overman EL, Stahl C, Moeser AJ. Early weaning stress in pigs impairs innate mucosal immune responses to enterotoxigenic E. coli challenge and exacerbates intestinal injury and clinical disease. PloS one. 2013;8(4):e59838.

46. Hui Q, Ammeter E, Liu S, Yang R, Lu P, Lahaye L, et al. Eugenol attenuates inflammatory response and enhances barrier function during lipopolysaccharide-induced inflammation in the porcine intestinal epithelial cells. J Anim Sci. 2020;98(8):skaa245.

47. Miller MB, Bassler BL. Quorum sensing in bacteria. Microbiology. 2001;55(1):165-99. 
48. Bai AJ, Rai VR. Bacterial quorum sensing and food industry. Compr Rev Food Sci F. 2011;10(3):18393.

49. Xavier KB, Bassler BL. LuxS quorum sensing: more than just a numbers game. Curr Opin Microbiol. 2003;6(2):191-7.

50. Schauder S, Shokat K, Surette MG, Bassler BL. The LuxS family of bacterial autoinducers: biosynthesis of a novel quorum-sensing signal molecule. Mol Microbiol. 2001;41(2):463-76.

51. Devi KR, Srinivasan S, Ravi AV. Inhibition of quorum sensing-mediated virulence in Serratia marcescens by Bacillus subtilis R-18. Microb Pathogenesis. 2018;120:166-75.

52. Kawai T, Akira S. The roles of TLRs, RLRs and NLRs in pathogen recognition. Int Immunol. 2009;21(4):317-37.

53. Kieser KJ, Kagan JC. Multi-receptor detection of individual bacterial products by the innate immune system. Nat Rev Immunol. 2017;17(6):376.

54. Qiao J, Sun Z, Liang D, Li H. Lactobacillus salivarius alleviates inflammation via NF-KB signaling in ETEC K88-induced IPEC-J2 cells. J Anim Sci Biotechno. 2020;11(1):1-13.

55. Wang X, Gao X, Hardwidge PR. Heat-labile enterotoxin-induced activation of NF-KB and MAPK pathways in intestinal epithelial cells impacts enterotoxigenic Escherichia coli (ETEC) adherence. Cell Microbiol. 2012;14(8):1231-41.

56. Chutkan $\mathrm{H}$, Kuehn M. Context-dependent activation kinetics elicited by soluble versus outer membrane vesicle-associated heat-labile enterotoxin. Infect Immun. 2011;79(9):3760-9.

\section{Figures}




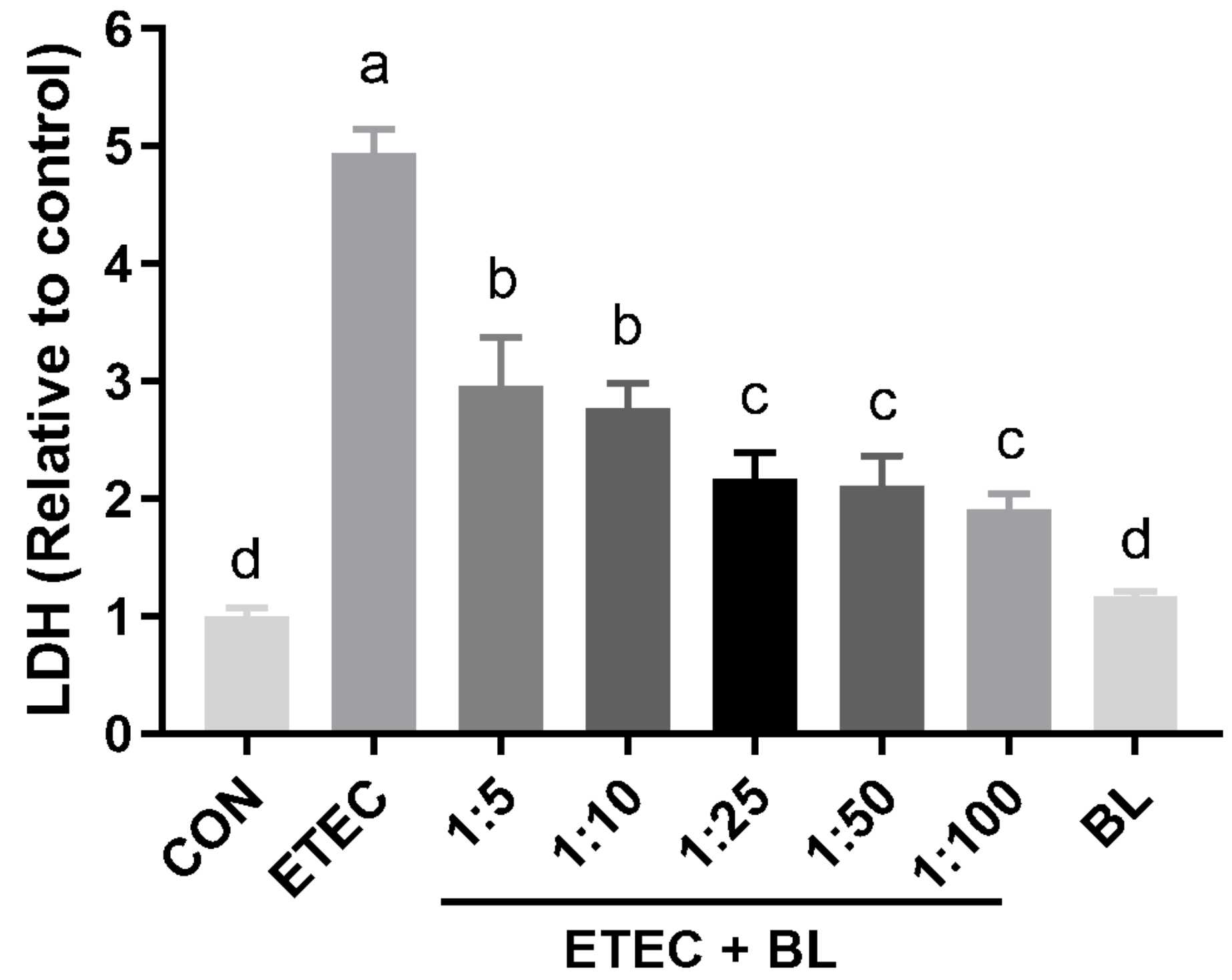

Figure 1

B. licheniformis PF9 protected IPEC-J2 cells against the ETEC F4-induced cytotoxicity. LDH activity in the supernatant was detected by LDH detection kit. The CON group was with no treatment. The groups of ETEC and BL were treated with $106 \mathrm{CFU} / \mathrm{mL}$ ETEC F4 alone and $108 \mathrm{CFU} / \mathrm{mL}$ B. licheniformis PF9 alone for $3 \mathrm{~h}$, respectively. The group of ETEC + BL were treated with both ETEC F4 (106 CFU/mL) and B. licheniformis PF9 at the ratio of 1:5, 1:10, 1:25, 1:50, or 1:100 (i.e., $5 \times 106,107,2.5 \times 107,5 \times 107$, or 108 $\mathrm{CFU} / \mathrm{mL}$ ) for $3 \mathrm{~h}$, respectively. The data were presented as mean $\pm S D, N=4$. Different letters represent a significant difference $(P<0.05)$. 


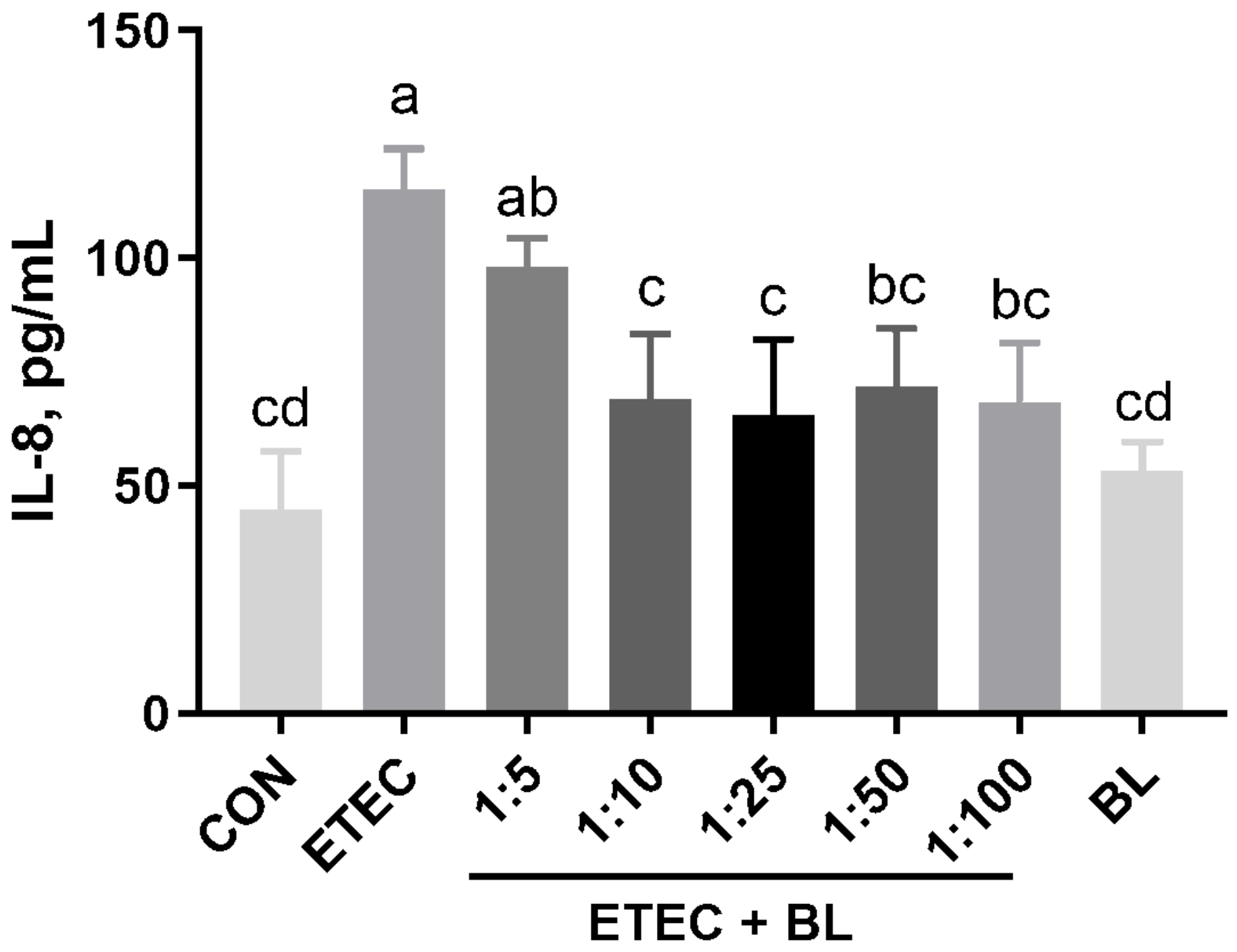

Figure 2

Effect of B. licheniformis PF9 on ETEC F4-induced the alteration of cytokine IL-8 secretion in IPEC-J2 cells. The treatments of these groups are the same as the above in Figure 1. The IL-8 concentration in the supernatant was measured by ELISA assay. The data were presented as mean $\pm S D, N=4$. Different letters represent a significant difference $(P<0.05)$. 
A

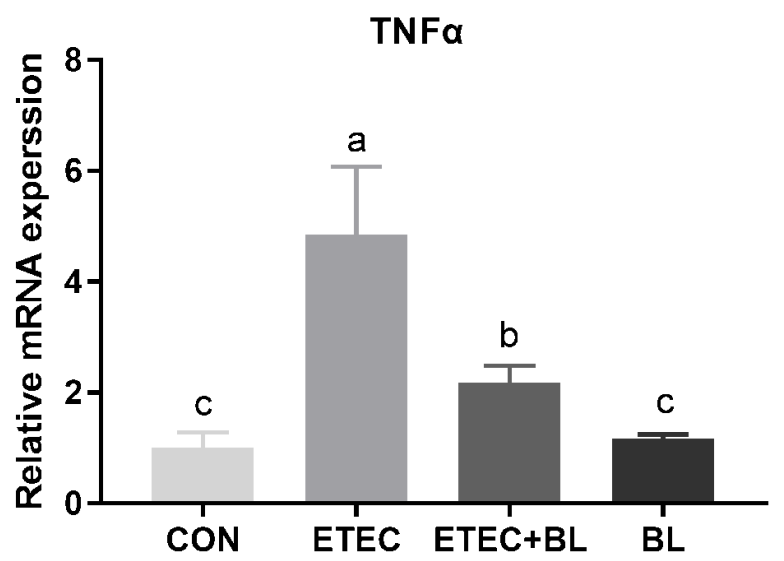

B

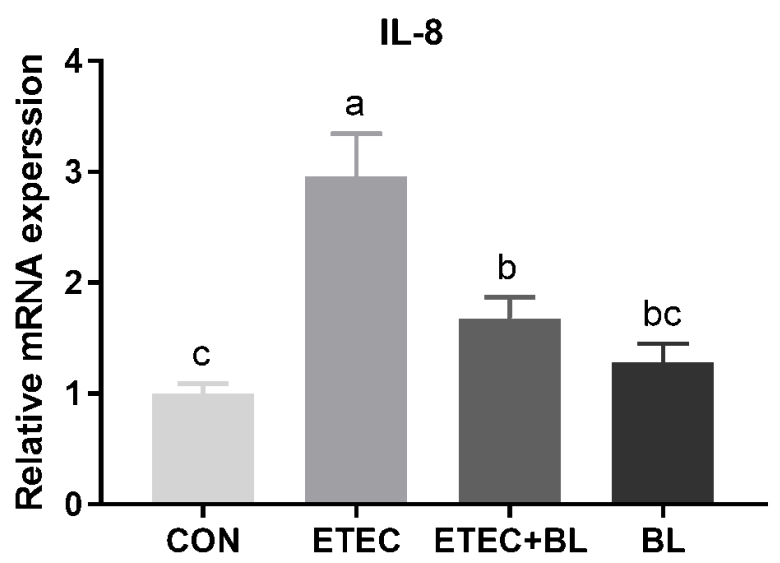

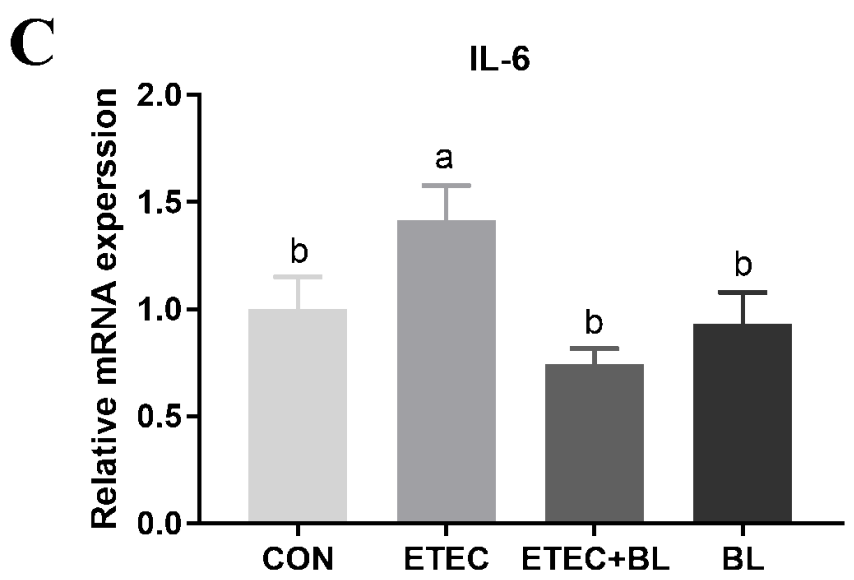

\section{Figure 3}

Effect of B. licheniformis PF9 on ETEC F4-induced gene expression of inflammatory cytokines in IPEC-J2 cells. The CON group was with no treatment. The groups of ETEC, ETEC + BL, and BL were treated with $106 \mathrm{CFU} / \mathrm{mL}$ ETEC F4 alone, both ETEC F4 (106 CFU/mL) and B. licheniformis PF9 (107 CFU/mL), and $107 \mathrm{CFU} / \mathrm{mL}$ B. licheniformis PF9 alone for $3 \mathrm{~h}$, respectively. These treatment groups were also used in the following Figures. Total RNA was extracted from IPEC-J2 cells and the mRNAs abundance of TNF-a (a), IL-8 (b), and IL-6 (c) were detected by qPCR. The data were presented as mean $\pm S D, N=4$. Different letters represent a significant difference $(P<0.05)$. 
$\mathbf{A}$

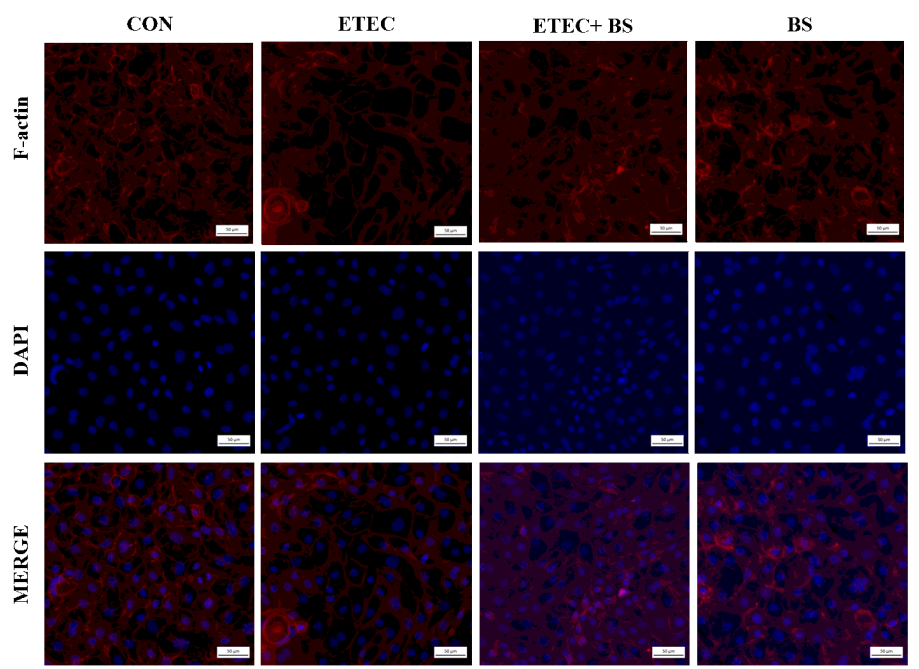

C
B
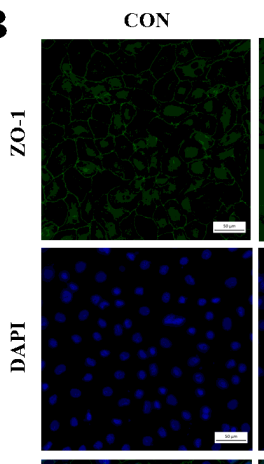

ETEC

ETEC+ BS

BS

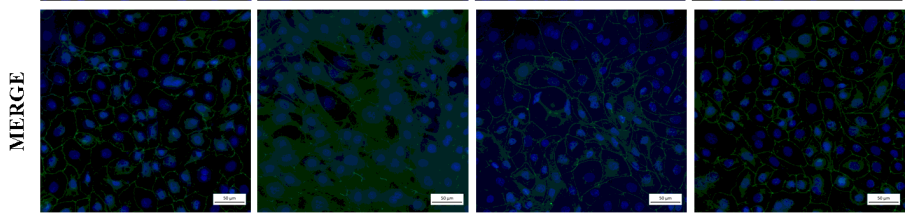

BS

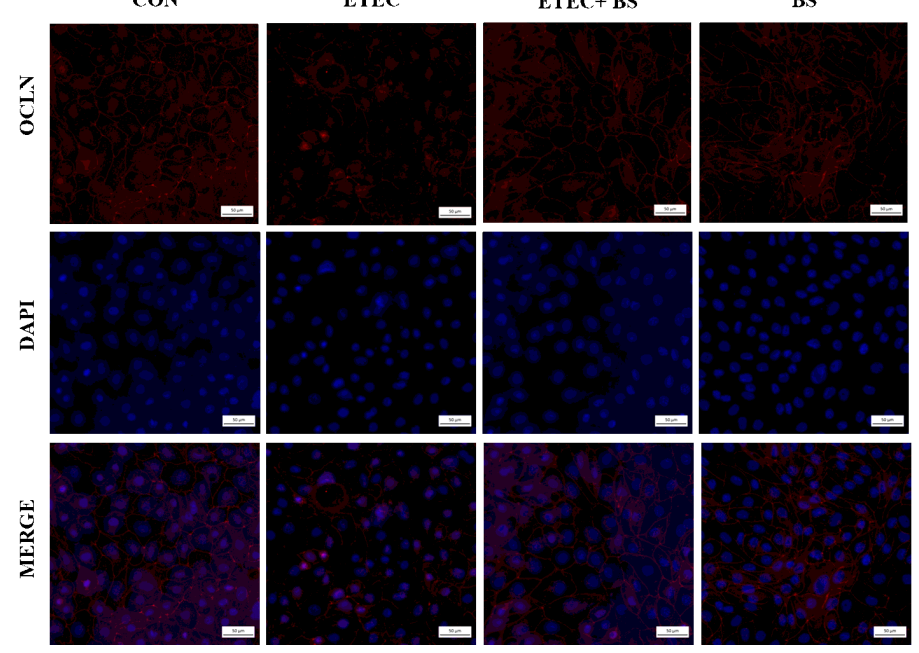

Figure 4

Protective effect of B. licheniformis PF9 against ETEC F4-induced morphological changes of cytoskeleton (F-actin, a) and TJs (ZO-1 and OCLN, b and c) in IPEC-J2 cells. 
A
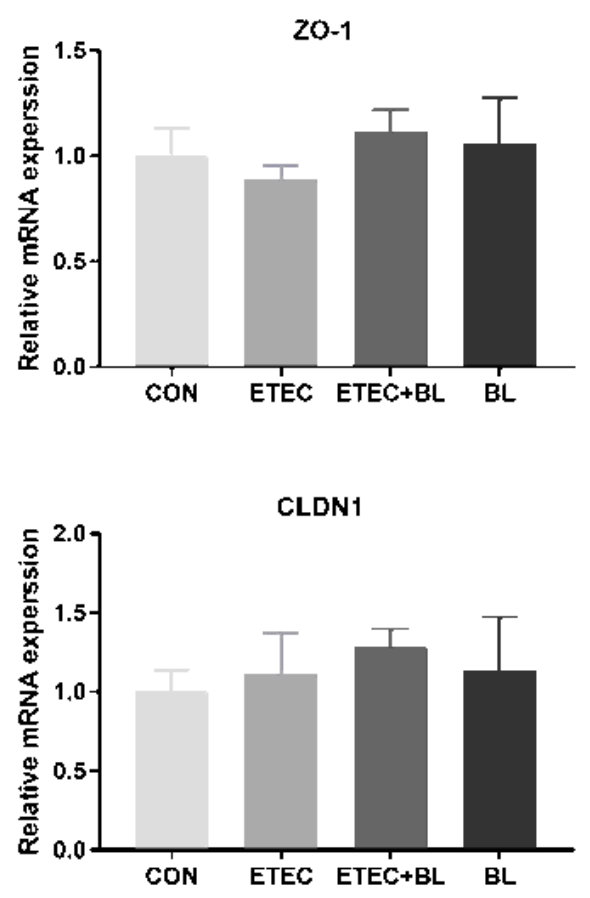

B
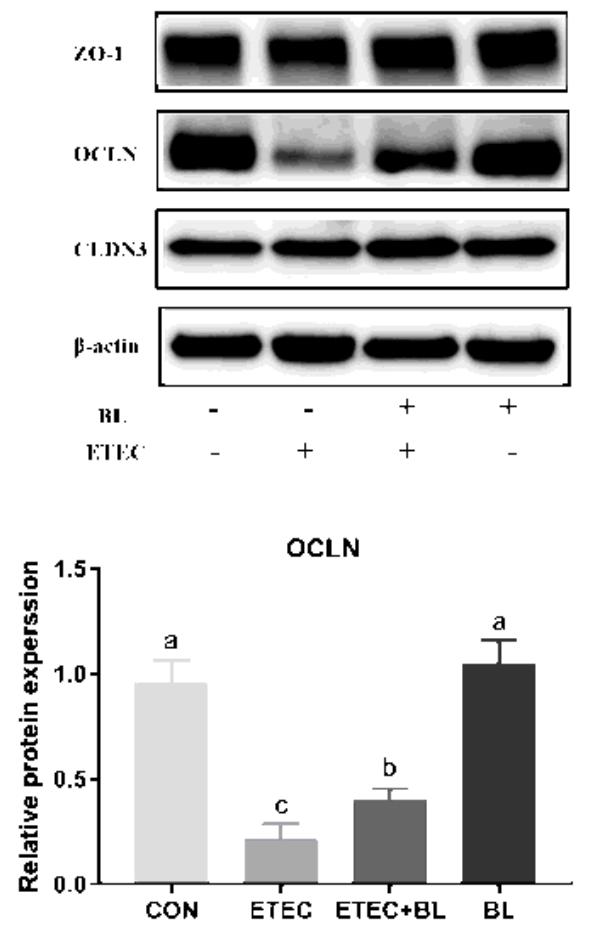
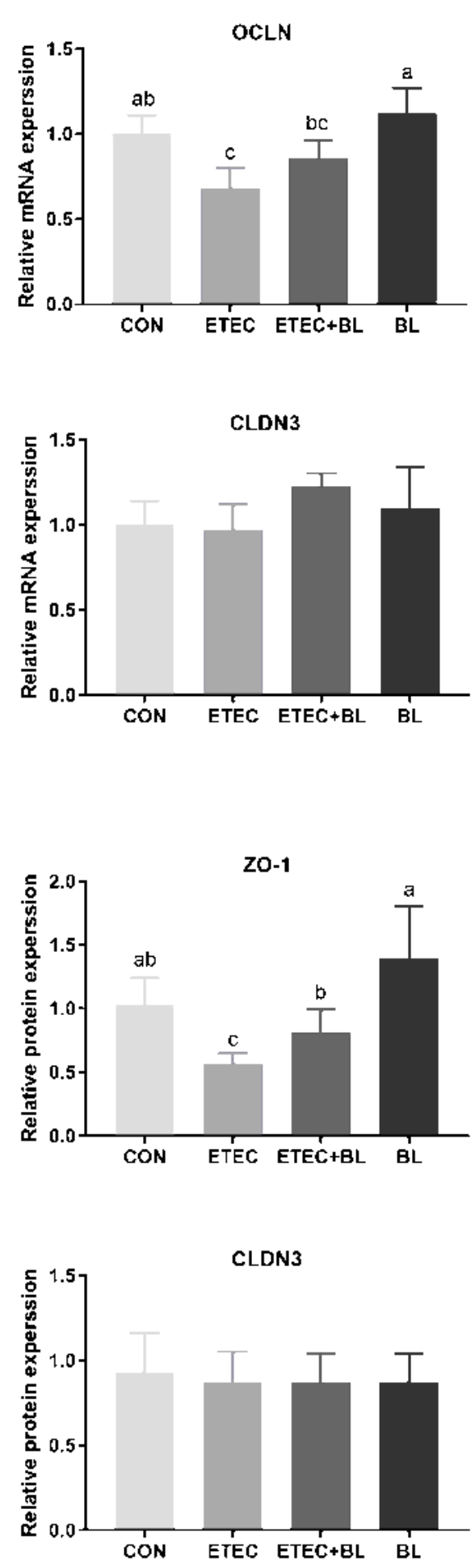

\section{Figure 5}

Influence of B. licheniformis PF9 on ETEC F4-induced gene and protein expressions of tight junctions (TJs) in IPEC-J2 cells. (a) Total RNA was extracted from IPEC-J2 cells and the gene expressions of ZO-1, OCLN, CLDN1, and CLDN3 were detected by qPCR $(\mathrm{N}=4)$. (b) Total protein was extracted from IPEC-J2 cells and the protein expressions of ZO-1, OCLN, and CLDN3 were detected by western blot $(\mathrm{N}=3)$. The data were presented as mean \pm SD. Different letters represent a significant difference $(P<0.05)$. 
A
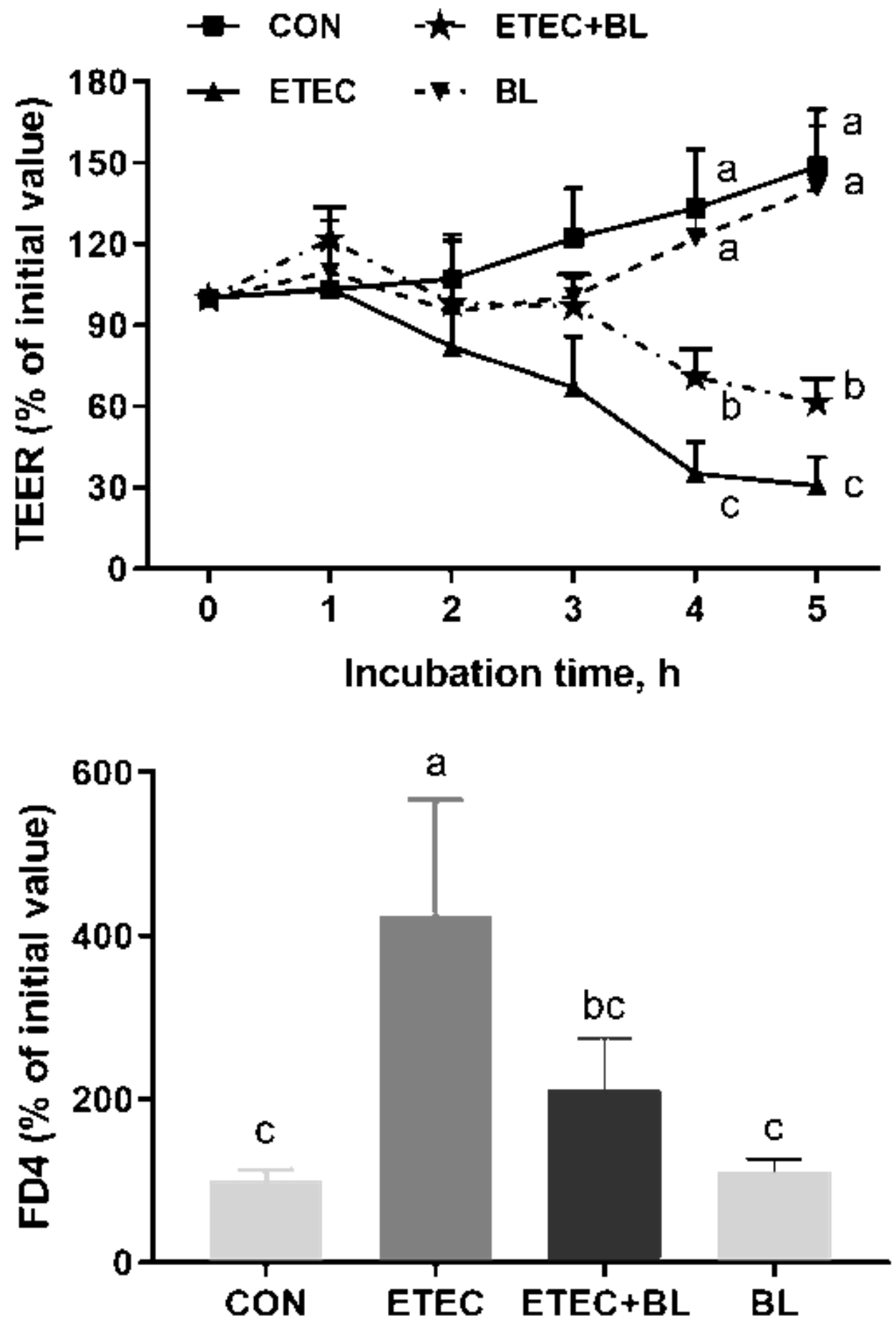

Figure 6

Effect of B. licheniformis PF9 on TEER and permeability in ETEC F4-challenged IPEC-J2 cells. (a) These TEER values were measured before $(0 \mathrm{~h})$ and after ETEC F4 treatments $(1,2,3,4$, and $5 \mathrm{~h}$ ). (b) Cell permeability was tested by the fluorescent intensity of FD4 after B. licheniformis PF9 treatment for $5 \mathrm{~h}$. The data were presented as mean $\pm S D, N=4$. Different letters indicate a significant difference at $P<$ 0.05 . 
A

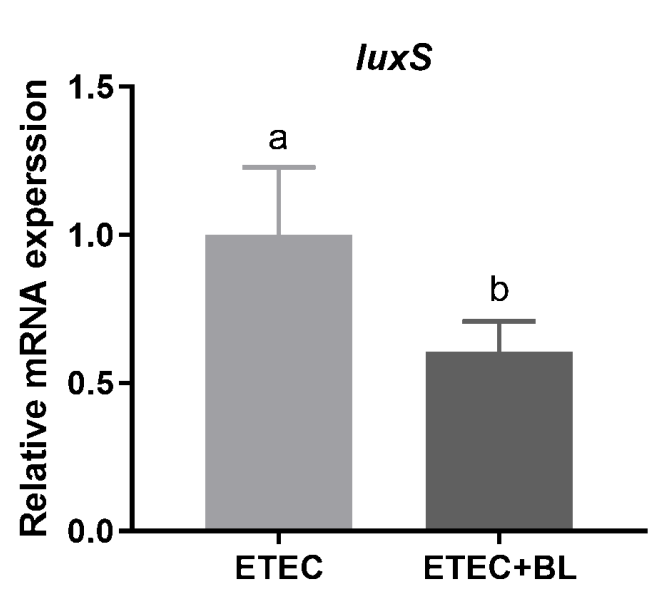

C

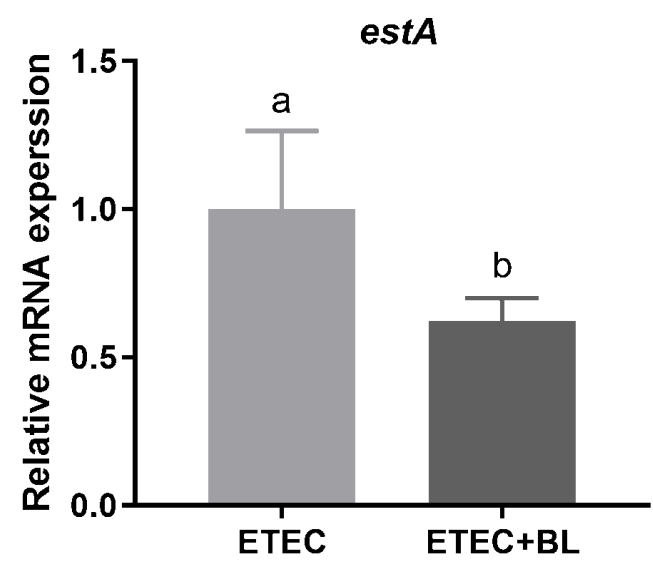

B

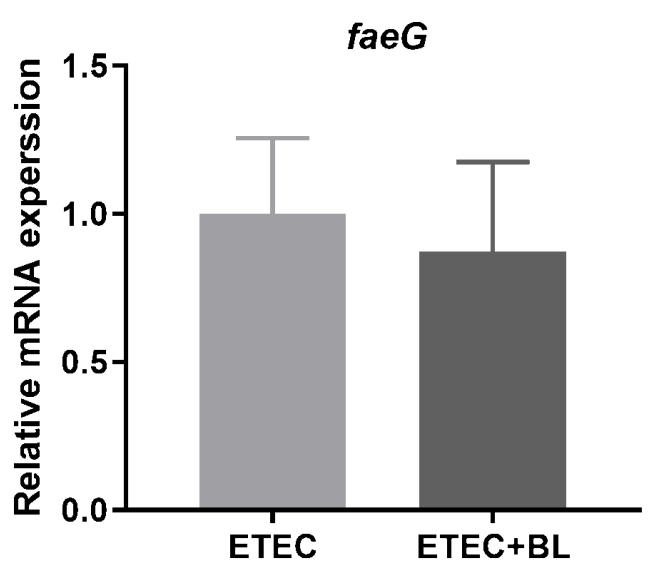

D

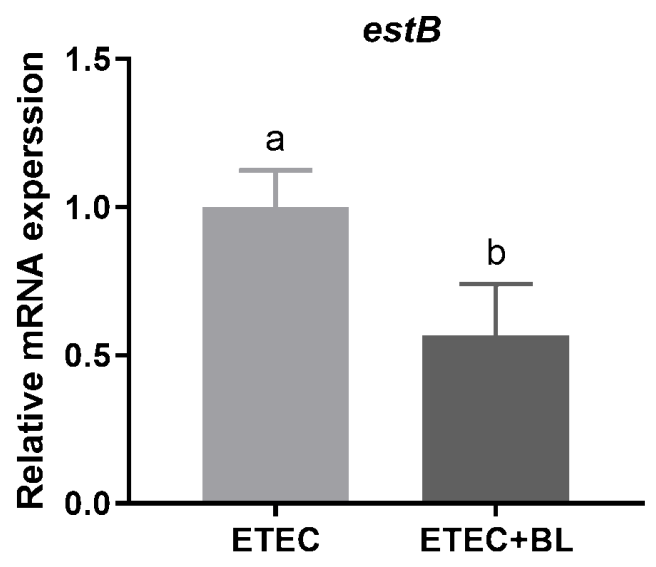

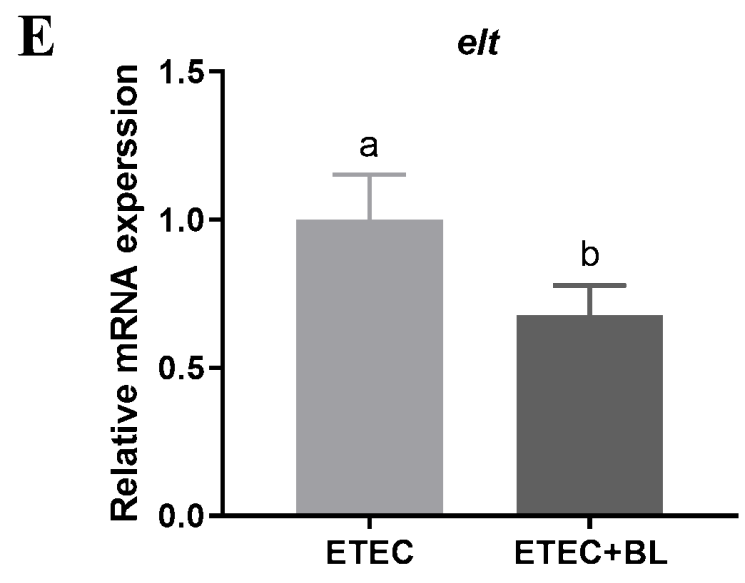

Figure 7

Effect of B. licheniformis PF9 on the mRNA abundance of virulence-related factors in ETEC F4. Total RNA was extracted from ETEC F4 and the mRNAs abundance of luxS (a), faeG (b), estA (c), estB (d), and elt (e) were detected by qPCR. The data were presented as mean $\pm S D, N=4$. Different letters represent a significant difference $(P<0.05)$. 
A
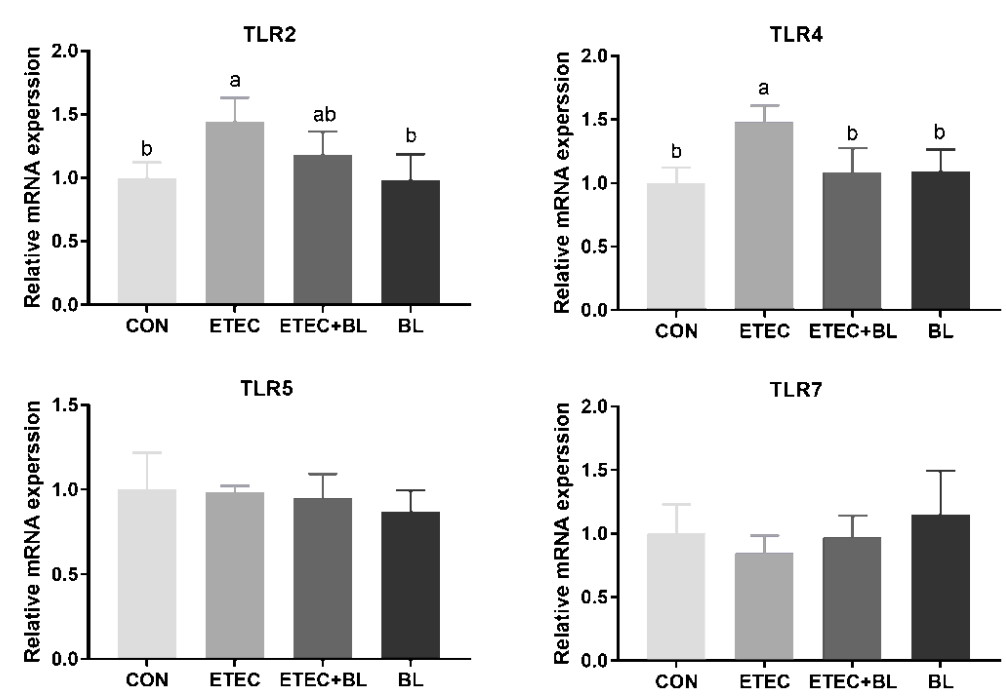

B
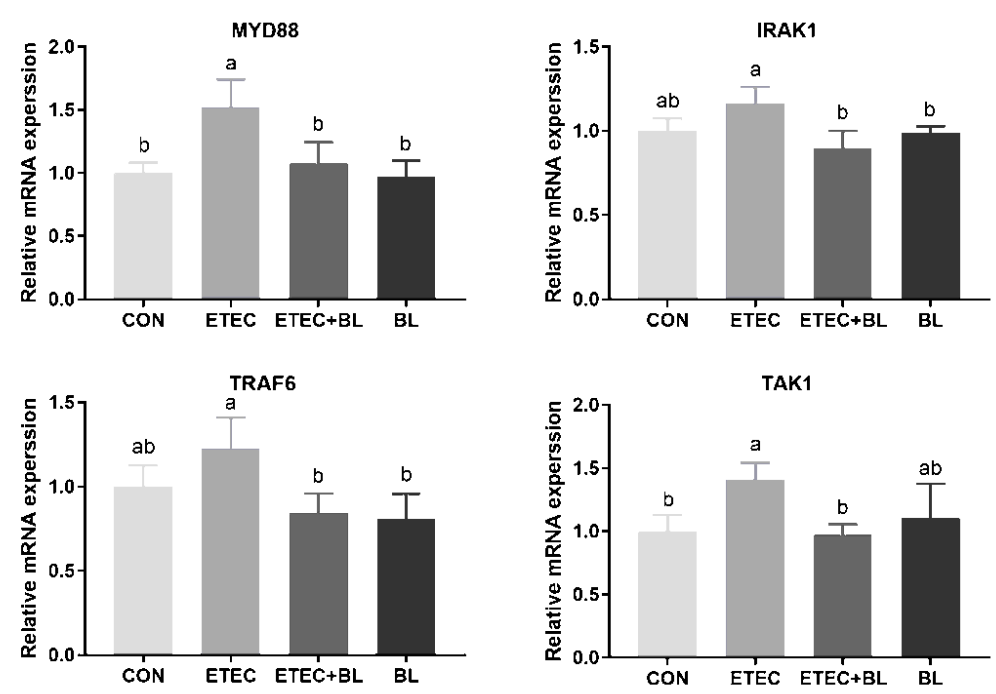

C
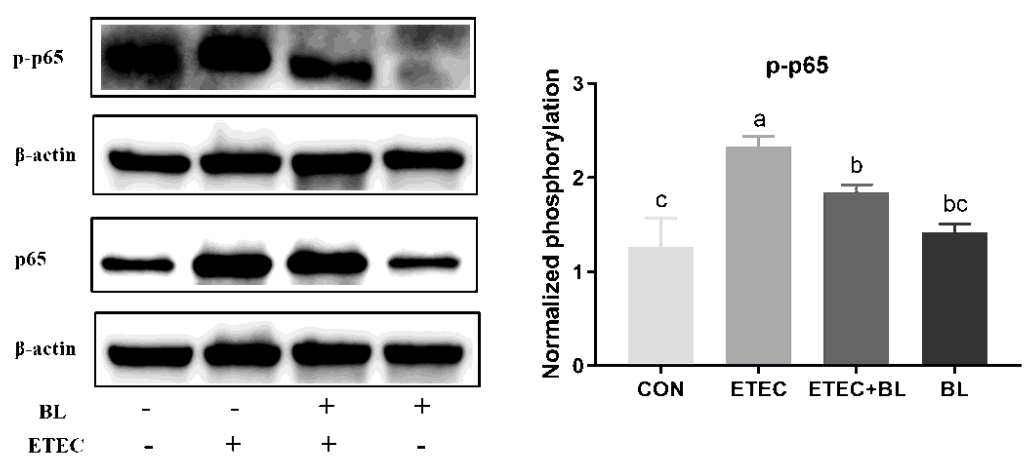

\section{Figure 8}

Effect of B. licheniformis PF9 on TLRs-mediated activation of the NF-KB signaling pathway after ETEC F4 stimulation in IPEC-J2 Cells. (a) The mRNA abundance of toll-like receptors was detected by qPCR ( $\mathrm{N}=$ 4). (b) The mRNA abundance of the related upstream genes of the NF-KB signaling pathway was detected by qPCR $(\mathrm{N}=4)$. (c) The protein abundances of p65 and p-p65 were detected by western blot ( $\mathrm{N}$ $=3)$. The data were presented as mean \pm SD. Different letters represent a significant difference $(P<0.05)$. 


\section{Supplementary Files}

This is a list of supplementary files associated with this preprint. Click to download.

- SupplementalMaterialsTable.docx 